\title{
Patient-controlled Epidural Analgesia: analysis of efficacy and complications in surgical oncology patients
}

Joana Sousa Correia' ${ }^{1}$ Mónica Nunes Ferreira ${ }^{1}$, Ana Afonso ${ }^{1}$, Mercedes Gacio ${ }^{1}$

${ }^{1}$ Department of Anaesthesiology and Intensive Care, Instituto Português de Oncologia, Porto, Portugal

\section{INTRODUCTION}

Patient-controlled epidural analgesia (PCEA) is a well established technique for postoperative pain relief after major surgery but several risks have been observed [1].

\section{PURPOSE}

The purpose of this study is to survey the efficacy and safety of PCEA and determining incidence, type and severity of postoperative complications related to epidural analgesia and catheter insertion.

\section{METHODS} center.

A retrospective study was conducted between May-September 2016 in a single-

- $\quad$ Patients were assessed by an acute pain service team postoperatively.

- The following variables were analyzed:

- type of surgery,

- incidence of side-effects/complications

- quality of analgesia (qualitative scale pain of 5 items: no, mild, moderate, severe, unbearable). We have considered as good analgesia only no/mild pain situations, reasonable analgesia when presented at least one episode of moderate pain, and poor analgesia at least one episode of severe/unbearable pain.

- We used SPSS (PSAW Statistics 18) for statistical analysis, non-parametric tests were performed, and a value of $p<0.05$ defined as a statistically significant result.

\section{RESULTS/DISCUSSION}

- Epidural catheters remained in place 4.63 days in average.

- Global rate of technique-related complications were $33.2 \%$, most of them minor complications without clinical repercussion (Graphic 1).

- From these $4.4 \%$ were directly related to the epidural catheter (disconnection, externalization, obstruction, leak of local anesthetic and machine fault).

- The most frequent complications were vomiting $(9.6 \%)$ and pruritus( $8 \%)$. Neurological complications represented $6.4 \%(6 \%$ paresthesias, $0.4 \%$ motor block) and cardiovascular complications $0,4 \%$ (hypotension). We also observed epidural failure(2.8\%), urinary retention $(0.8 \%)$, postdural puncture headache $(0.4 \%)$ and inflammation and local infection(0.4\%) (Graphic 2).

- Postoperative analgesia was effective in the majority of the patients. Assessment of resting/movement analgesia: good in $97.2 \% / 76.4 \%$; reasonable in $2 \% / 21.2 \%$; poor in $0.8 \% / 2.4 \%$ (Graphic 3).
- 250 elective surgical patients

- 250 included in the study

\section{PCEA}

\section{$-0,1 \%$ Ropivacaine + Sufentanil} $0.5 \mu \mathrm{g} / \mathrm{ml}$

- Rate: $5-15 \mathrm{ml}^{\mathrm{h}} \mathrm{h}^{-1}$

- Loading dose: $5-15 \mathrm{ml}$

- Possibility of $3-5 \mathrm{ml}$ bolus (interval of 20min).

- Maximum dose in 4hours:

- 60ml: thoracic epidural

- 80ml: lumbar epidural

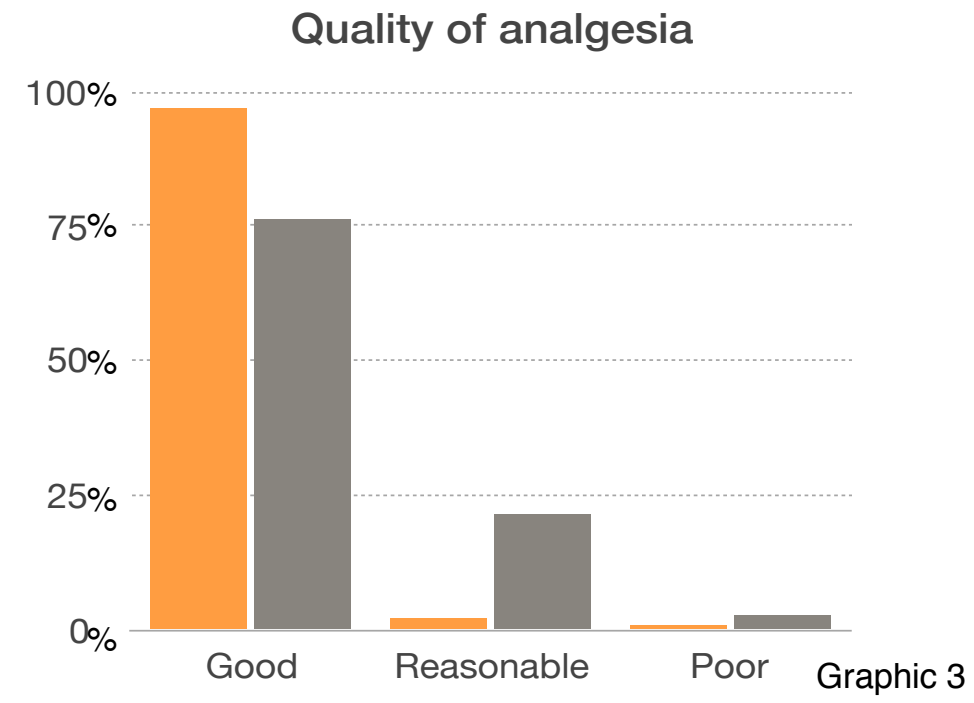

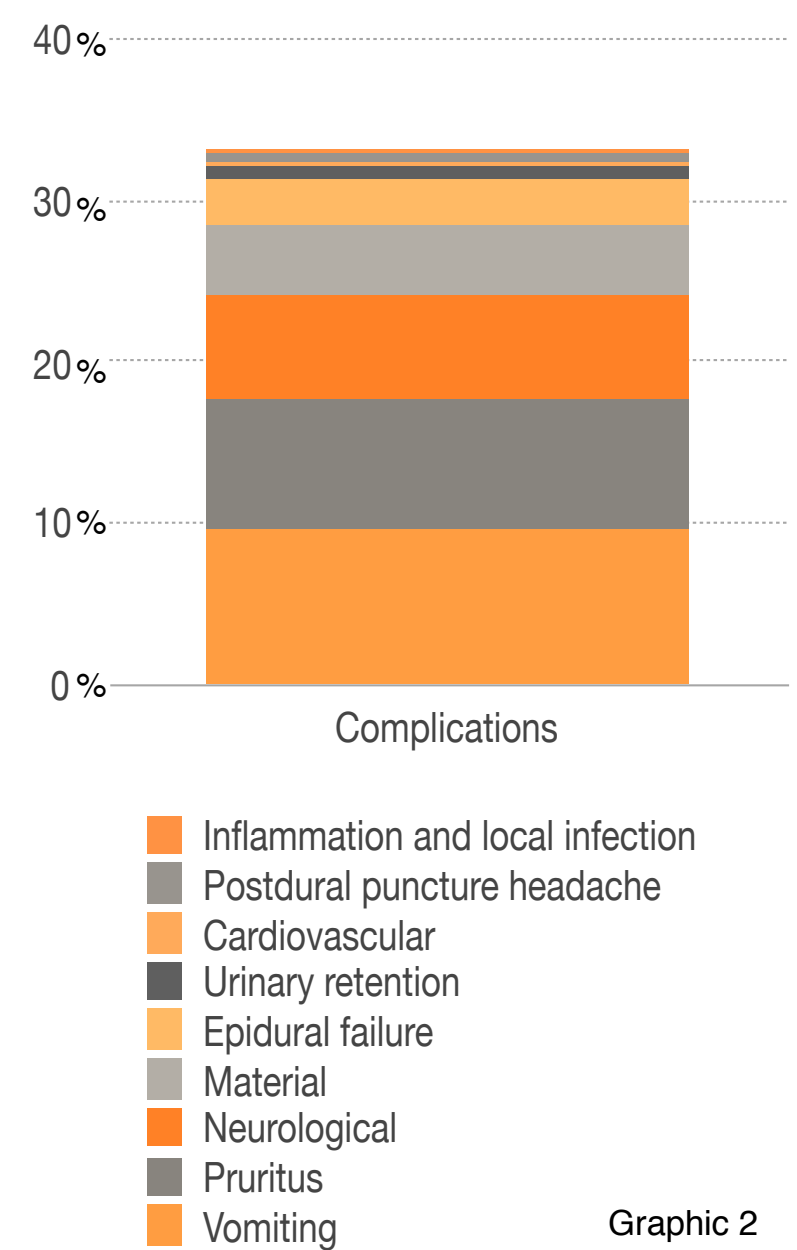

Graphic 2

\section{CONCLUSION}

PCEA was efective and safe in the postoperative period. Complications were few and not severe. A strict vigilance is recommended to achieve satisfactory analgesia and a low incidence of complications.

\section{REFERENCES}

1. Korean J Pain $201326: 39-45$ 